\title{
A note on the boundedness of sublinear operators on grand variable Herz spaces
}

\author{
Hammad Nafis ${ }^{1}$, Humberto Rafeiro ${ }^{2^{*}}$ (I) and Muhammad Asad Zaighum ${ }^{1}$
}

\section{"Correspondence:}

rafeiro@uaeu.ac.ae

${ }^{2}$ College of Sciences, Department of Mathematical Sciences, United Arab Emirates University, Al Ain, United Arab Emirates

Full list of author information is available at the end of the article

\section{Springer}

\begin{abstract}
In this paper, we introduce grand variable Herz type spaces using discrete grand spaces and prove the boundedness of sublinear operators on these spaces.

MSC: 46E30

Keywords: Sublinear operators; Herz spaces; Variable exponent analysis; Grand spaces
\end{abstract}

\section{Introduction}

The Herz spaces $\dot{K}_{q}^{\alpha, p}\left(\mathbb{R}^{n}\right)$ and $K_{q}^{\alpha, p}\left(\mathbb{R}^{n}\right)$ were introduced in [10] being known under the names of homogeneous and non-homogeneous Herz spaces and they are defined by the norms

$$
\begin{aligned}
& \|f\|_{\dot{K}_{q}^{\alpha, p}}:=\left\{\sum_{k \in \mathbb{Z}} 2^{k \alpha p}\left(\int_{R_{2^{k-1}, 2^{k}}}|f(x)|^{q} d x\right)^{p / q}\right\}^{1 / p}, \\
& \|f\|_{K_{q}^{\alpha, p}}:=\|f\|_{L^{q}(B(0.1))}+\left\{\sum_{k \in \mathbb{N}} 2^{k \alpha p}\left(\int_{R_{2^{k-1,2^{k}}}}|f(x)|^{q} d x\right)^{p / q}\right\}^{1 / p},
\end{aligned}
$$

respectively, where $R_{t, \tau}$ stands for the annulus $R_{t, \tau}:=B(0, \tau) \backslash B(0, t)$. These spaces were studied in many papers; see for instance $[5,7,9,12-15,22]$ and the references therein.

Last two decades, under the influence of some applications revealed in [32], there was a vast boom of research in the so called variable exponent spaces (see e.g. [30]). For the time being, the theory of such variable exponent Lebesgue, Orlicz, Lorentz, and Sobolev function spaces is widely developed, we refer to the books [2-4,20,21]. Herz spaces with variables exponent have been recently introduced in $[1,12,13]$. Samko in [33] used variable exponent Herz spaces (with variable parameters), known as continual Herz spaces. Another approach regarding variable smoothness and integrability to study Herz type Hardy spaces was used in [29].

Grand Lebesgue spaces on bounded sets have been widely studied. They were introduced in $[8,11]$, cf. [2]. Grand spaces proved to be useful in application to partial differential equations. Various operators of harmonic analysis were intensively studied in the last years, cf. $[6,16-20,27,28]$ and the references therein.

c) The Author(s) 2019. This article is licensed under a Creative Commons Attribution 4.0 International License, which permits use, sharing, adaptation, distribution and reproduction in any medium or format, as long as you give appropriate credit to the original author(s) and the source, provide a link to the Creative Commons licence, and indicate if changes were made. The images or other third party material in this article are included in the article's Creative Commons licence, unless indicated otherwise in a credit line to the material. If material is not included in the article's Creative Commons licence and your intended use is not permitted by statutory regulation or exceeds the permitted use, you will need to obtain permission directly from the copyright holder. To view a copy of this licence, visit http://creativecommons.org/licenses/by/4.0/. 
Grand Lebesgue sequence spaces were introduced in [31], where various operators of harmonic analysis were studied in these spaces, e.g. maximal, convolutions, Hardy, Hilbert, and fractional operators, among others.

The aim of this paper is to introduce grand variable Herz spaces $\dot{K}_{q}^{\alpha, p), \theta}\left(\mathbb{R}^{n}\right)$ and obtain the boundedness of sublinear operators on $\dot{K}_{q}^{\alpha, p), \theta}\left(\mathbb{R}^{n}\right)$. The present article has three sections apart from the Introduction. Section 2 deals with some basic notions regarding grand Lebesgue sequence spaces. In Sect. 3 we give the definition of grand variable Herz spaces and prove Hölder's inequality. In Sect. 4 we discuss boundedness of sublinear operators on grand variable Herz spaces. Throughout the paper, constants (often different constant in the same series of inequalities) will mainly be denoted by $c$ or $C$. $f \lesssim g$ means that $f \leq C g$ and $f \approx g$ means that $f \lesssim g \lesssim f$.

\section{Preliminaries}

\subsection{Lebesgue space with variable exponent}

For the current section we refer to $[4,23]$ unless and until stated otherwise. Let $X \subseteq \mathbb{R}^{n}$ be an open set and $p(\cdot)$ be a real-valued measurable function on $X$ with values in $[1, \infty)$. We suppose that

$$
1 \leq p_{-}(X) \leq p_{+}(X)<\infty
$$

where $p_{-}(X):=\operatorname{essinf}_{x \in X} p(x)$ and $p_{+}(X):=\operatorname{ess} \sup _{x \in X} p(x)$. By $L^{p(\cdot)}(X)$ we denote the space of measurable function $f$ on $X$ such that

$$
I_{p(\cdot)}(f)=\int_{X}|f(x)|^{p(x)} d x<\infty .
$$

It is a Banach space equipped with the norm (see e.g. [4]):

$$
\|f\|_{L^{p(\cdot)(X)}}=\inf \left\{\eta>0: I_{p(\cdot)}\left(\frac{f}{\eta}\right) \leq 1\right\} .
$$

By $p^{\prime}(x)=p(x) /(p(x)-1)$, we denote the conjugate exponent of $p(\cdot)$. For the following lemma we refer to e.g. [3].

Lemma 2.1 (Generalized Hölder's inequality) Let $X$ be a measurable subset of $\mathbb{R}^{n}$. Suppose that $1 \leq p_{-}(X) \leq p_{+}(X)<\infty$. Then

$$
\|f g\|_{L^{r \cdot(\cdot)}(X)} \leq c\|f\|_{L^{p \cdot \cdot(X)}}\|g\|_{L^{q(\cdot)}(X)}
$$

holds, where $f \in L^{p(\cdot)}(X), g \in L^{q(\cdot)}(X)$ and $\frac{1}{r(x)}=\frac{1}{p(x)}+\frac{1}{q(x)}$ for every $x \in X$.

In the sequel we use the well known log-condition

$$
|q(x)-q(y)| \leq \frac{A}{-\ln |x-y|}, \quad|x-y| \leq \frac{1}{2}, x, y \in X,
$$

where $A=A(q)>0$ does not depend on $x, y$, and the decay condition: there exists a number $q_{\infty} \in(1, \infty)$, such that

$$
\left|q(x)-q_{\infty}\right| \leq \frac{A}{\ln (e+|x|)},
$$


and also the decay condition

$$
\left|q(x)-q_{0}\right| \leq \frac{A}{\ln |x|}, \quad|x| \leq \frac{1}{2},
$$

holds for some $q_{0} \in(1, \infty)$ in the case of homogeneous Herz spaces.

With respect to classes of variable exponents used in this paper, we adopt the following notations:

(i) Given a function $f \in L_{\mathrm{loc}}^{1}(X)$, the Hardy-Littlewood maximal operator $M$ is defined by

$$
M f(x):=\sup _{r>0} r^{-n} \int_{B(x, r)}|f(y)| d y \quad(x \in X),
$$

where

$$
B(x, r):=\{y \in X:|x-y|<r\} .
$$

(ii) $L_{\mathrm{loc}}^{q(\cdot)}(X):=\left\{f: f \in L^{q(\cdot)}(K)\right.$ for all compact subsets $\left.K \subset X\right\}$.

(iii) The set $\mathcal{P}(X)$ consists of all $q(\cdot)$ satisfying $q_{-}>1$ and $q_{+}<\infty$.

(iv) $\mathcal{P}^{\log }=\mathcal{P}^{\log }(X)$ is the class of functions $q \in \mathcal{P}(X)$ satisfying the conditions (3) and (4).

(v) In the case $X$ is unbounded, $\mathcal{P}_{\infty}(X)$ and $\mathcal{P}_{0, \infty}(X)$ are subsets of exponents in $\mathcal{P}(X)$ with values in $[1, \infty)$ which satisfy condition (5) and the two conditions (5) and (6), respectively.

(vi) $\mathcal{B}(X)$ is the set of $q(\cdot) \in \mathcal{P}(X)$ satisfying the condition that $M$ is bounded on $L^{q(\cdot)}(X)$.

The following lemma appears in [33].

Lemma 2.2 Let $D>1$ and $q \in \mathcal{P}_{0, \infty}\left(\mathbb{R}^{n}\right)$. Then

$$
\frac{1}{c_{0}} r^{\frac{n}{q(0)}} \leq\left\|\chi_{R_{r, D r}}\right\|_{q(\cdot)} \leq c_{0} r^{\frac{n}{q(0)}}, \quad \text { for } 0<r \leq 1,
$$

and

$$
\frac{1}{c_{\infty}} r^{\frac{n}{q \infty}} \leq\left\|\chi_{R_{r, D r}}\right\|_{q(\cdot)} \leq c_{\infty} r^{\frac{n}{q \infty}}, \quad \text { for } r \geq 1,
$$

respectively, where $c_{0} \geq 1$ and $c_{\infty} \geq 1$ depend on $D$, but do not depend on $r$.

\subsection{Herz spaces with variable exponent}

In this subsection, we introduce variable exponent Herz spaces. In what follows, we denote $\chi_{k}=\chi_{R_{k}}, R_{k}=B_{k} \backslash B_{k-1}$ and $B_{k}=\left\{x \in \mathbb{R}^{n}:|x| \leq 2^{k}\right\}$ for all $k \in \mathbb{Z}$.

Definition 2.1 Let $1<p<\infty, \alpha \in \mathbb{R}$ and $q(\cdot) \in \mathcal{P}\left(\mathbb{R}^{n}\right)$. The homogeneous Herz space $\dot{K}_{q(\cdot)}^{\alpha, p}\left(\mathbb{R}^{n}\right)$ is defined by

$$
\dot{K}_{q(\cdot)}^{\alpha, p}\left(\mathbb{R}^{n}\right)=\left\{f \in L_{\mathrm{loc}}^{q(\cdot)}\left(\mathbb{R}^{n} \backslash\{0\}\right):\|f\|_{\dot{K}_{q(\cdot)}^{\alpha, p}\left(\mathbb{R}^{n}\right)}<\infty\right\},
$$


where

$$
\|f\|_{\dot{K}_{q(\cdot)}^{\alpha, p}\left(\mathbb{R}^{n}\right)}=\left(\sum_{k=-\infty}^{\infty}\left\|2^{k \alpha} f \chi_{k}\right\|_{L^{q(\cdot)}}^{p}\right)^{\frac{1}{p}} .
$$

Definition 2.2 Let $1<p<\infty, \alpha \in \mathbb{R}$ and $q(\cdot) \in \mathcal{P}\left(\mathbb{R}^{n}\right)$. The non-homogeneous Herz space $K_{q(\cdot)}^{\alpha, p}\left(\mathbb{R}^{n}\right)$ is defined by

$$
K_{q(\cdot)}^{\alpha, p}\left(\mathbb{R}^{n}\right)=\left\{f \in L_{\text {loc }}^{q(\cdot)}\left(\mathbb{R}^{n}\right):\|f\|_{K_{q(\cdot)}^{\alpha, p}\left(\mathbb{R}^{n}\right)}<\infty\right\},
$$

where

$$
\|f\|_{K_{q(\cdot)}^{\alpha, p}\left(\mathbb{R}^{n}\right)}=\left(\sum_{k=1}^{\infty}\left\|2^{k \alpha} f \chi_{k}\right\|_{L^{q(\cdot)}}^{p}\right)^{\frac{1}{p}}+\|f\|_{L^{q(\cdot)} B(0,1)} .
$$

\subsection{Grand Lebesgue sequence space}

In this subsection we introduce grand Lebesgue sequence space. For the following definitions and statements, see [31]. The letter $\mathbb{X}$ stands for one of the sets $\mathbb{Z}^{n}, \mathbb{Z}, \mathbb{N}$ and $\mathbb{N}_{0}$.

Definition 2.3 Let $1 \leq p<\infty$ and $\theta>0$. The grand Lebesgue sequence space $l^{p), \theta}$ is defined by the norm

$$
\begin{aligned}
\left\|\left\{x_{k}\right\}_{k \in \mathbb{X}}\right\|_{p^{p), \theta}(\mathbb{X})} & =\|\mathbf{x}\|_{\left.l^{p}\right), \theta(\mathbb{X})} \\
& :=\sup _{\varepsilon>0}\left(\varepsilon^{\theta} \sum_{k \in \mathbb{X}}\left|x_{k}\right|^{p(1+\varepsilon)}\right)^{\frac{1}{p(1+\varepsilon)}}=\sup _{\varepsilon>0} \varepsilon^{\frac{\theta}{p(1+\varepsilon)}}\|x\|_{l^{p(1+\varepsilon)}(\mathbb{X})},
\end{aligned}
$$

where $\mathbf{x}=\left\{x_{k}\right\}_{k \in \mathbb{X}}$.

Note that the following nesting properties hold:

$$
l^{p(1-\varepsilon)} \hookrightarrow l^{p} \hookrightarrow l^{p), \theta_{1}} \hookrightarrow l^{p), \theta_{2}} \hookrightarrow l^{p(1+\delta)}
$$

for $0<\varepsilon<\frac{1}{p}, \delta>0$ and $0<\theta_{1} \leq \theta_{2}$.

\section{Grand variable Herz space}

In this section, we introduce grand variable Herz space in a natural way using the discrete space from Definition 2.3.

Definition 3.1 Let $\alpha \in \mathbb{R}, 1 \leq p<\infty, q: \mathbb{R}^{n} \rightarrow[1, \infty), \theta>0$. We define the homogeneous grand variable Herz space by

$$
\dot{K}_{q(\cdot)}^{\alpha, p), \theta}\left(\mathbb{R}^{n}\right)=\left\{f \in L_{\mathrm{loc}}^{q(\cdot)}\left(\mathbb{R}^{n} \backslash\{0\}\right):\|f\|_{\dot{K}_{q(\cdot)}^{\alpha, p), \theta}\left(\mathbb{R}^{n}\right)}<\infty\right\},
$$


where

$$
\begin{aligned}
& \|f\|_{\dot{K}_{q(\cdot)}^{\alpha, p), \theta}\left(\mathbb{R}^{n}\right)}=\sup _{\varepsilon>0}\left(\varepsilon^{\theta} \sum_{k \in \mathbb{Z}} 2^{k \alpha p(1+\varepsilon)}\left\|f \chi_{k}\right\|_{L^{q(\cdot)}}^{p(1+\varepsilon)}\right)^{\frac{1}{p(1+\varepsilon)}} \\
& =\sup _{\varepsilon>0} \varepsilon^{\frac{1}{p(1+\varepsilon)}}\|f\|_{\dot{K}_{q(\cdot)}^{\alpha, p(1+\varepsilon)}\left(\mathbb{R}^{n}\right)^{n}} .
\end{aligned}
$$

In a similar way, non-homogeneous grand variable Herz spaces can be introduced. In the following theorem, we prove that Herz space is contained in grand variable Herz space.

Theorem 3.1 For $p>1$, we have $\dot{K}_{q(\cdot)}^{\alpha, p}\left(\mathbb{R}^{n}\right) \subset \dot{K}_{q(\cdot)}^{\alpha, p), \theta}\left(\mathbb{R}^{n}\right), \theta>0$.

Proof Let $f \in \dot{K}_{q(\cdot)}^{\alpha, p}\left(\mathbb{R}^{n}\right)$. Then

$$
\begin{aligned}
\|f\|_{\dot{K}_{q(\cdot)}^{\alpha, p), \theta}\left(\mathbb{R}^{n}\right)} & =\sup _{\varepsilon>0}\left(\varepsilon^{\theta} \sum_{k \in \mathbb{Z}} 2^{k \alpha p(1+\varepsilon)}\left\|f \chi_{k}\right\|_{L^{q(\cdot)}}^{p(1+\varepsilon)}\right)^{\frac{1}{p(1+\varepsilon)}} \\
& =\left\|2^{k \alpha}\right\| f \chi_{k}\left\|_{L^{q(\cdot)}}\right\|_{\left.p^{p}\right), \theta} \\
& \leq C\left\|2^{k \alpha}\right\| f \chi_{k}\left\|_{L^{q(\cdot)}}\right\|_{l^{p}}=C\left(\sum_{k \in \mathbb{Z}} 2^{k \alpha p}\left\|f \chi_{k}\right\|_{L^{q(\cdot)}}^{p}\right)^{\frac{1}{p}} \\
& =C\|f\|_{\dot{K}_{q(\cdot)}^{\alpha, p}\left(\mathbb{R}^{n}\right)},
\end{aligned}
$$

where we used (9).

Now we prove the Hölder inequality for a grand variable Herz space.

Theorem 3.2 If $0<p_{i} \leq \infty, 1 \leq q_{-} \leq q_{+}<\infty,-\infty<\alpha_{i}<\infty, i=1,2, \frac{1}{p}=\frac{1}{p_{1}}+\frac{1}{p_{2}}$, $1=\frac{1}{q(\cdot)}+\frac{1}{q^{\prime}(\cdot)}$ and $\alpha=\alpha_{1}+\alpha_{2}$. Then

$$
\|f g\|_{\dot{K}_{1}^{\alpha, p), \theta}\left(\mathbb{R}^{n}\right)} \leq\|f\|_{\dot{K}_{q(\cdot)}^{\left.\alpha_{1}, p_{1}\right), \theta}\left(\mathbb{R}^{n}\right)}\|g\|_{\dot{K}_{q^{\prime}(\cdot)}^{\left.\alpha_{2}, p_{2}\right), \theta}\left(\mathbb{R}^{n}\right)^{*}} .
$$

Proof We have

$$
\begin{aligned}
\|f g\|_{\dot{K}_{1}^{\alpha, p), \theta}\left(\mathbb{R}^{n}\right)} & =\sup _{\varepsilon>0}\left(\varepsilon^{\theta} \sum_{k \in \mathbb{Z}} 2^{k \alpha p(1+\varepsilon)}\left\|f g \chi_{k}\right\|_{L^{1}\left(\mathbb{R}^{n}\right)}^{p(1+\varepsilon)}\right)^{\frac{1}{p(1+\varepsilon)}} \\
& =\sup _{\varepsilon>0}\left(\varepsilon^{\theta} \sum_{k \in \mathbb{Z}} 2^{k \alpha p(1+\varepsilon)}\left(\int_{2^{k}}^{2^{k+1}}|f g|\right)^{p(1+\varepsilon)}\right)^{\frac{1}{p(1+\varepsilon)}} .
\end{aligned}
$$

By using Hölder's inequality

$$
\begin{aligned}
& \leq C \sup _{\varepsilon>0}\left(\varepsilon^{\theta} \sum_{k \in \mathbb{Z}} 2^{k\left(\alpha_{1}+\alpha_{2}\right) p(1+\varepsilon)}\left(\left\|f \chi_{k}\right\|_{L^{q(\cdot)}}^{p(1+\varepsilon)}\right)\left(\left\|g \chi_{k}\right\|_{L^{q^{\prime}(\cdot)}}^{p(1+\varepsilon)}\right)\right)^{\frac{1}{p(1+\varepsilon)}} \\
& =C \sup _{\varepsilon>0}\left(\varepsilon^{\theta} \sum_{k \in \mathbb{Z}}\left(2^{k \alpha_{1}}\left\|f \chi_{k}\right\|_{L^{q(\cdot)}}\right)^{p(1+\varepsilon)}\left(2^{k \alpha_{2}}\left\|g \chi_{k}\right\|_{L^{q^{\prime}(\cdot)}}\right)^{p(1+\varepsilon)}\right)^{\frac{1}{p(1+\varepsilon)}},
\end{aligned}
$$


by generalized Hölder's inequality

$$
\begin{aligned}
\leq & \sup _{\varepsilon>0}\left(\varepsilon^{\theta}\left(\sum_{k \in \mathbb{Z}}\left(2^{k \alpha_{1}}\left\|f \chi_{k}\right\|_{L^{q(\cdot)}}\right)^{p_{1}(1+\varepsilon)}\right)^{1 / p_{1}(1+\varepsilon)}\right. \\
& \left.\times \sup _{\varepsilon>0}\left(\varepsilon^{\theta} \sum_{k \in \mathbb{Z}}\left(2^{k \alpha_{2}}\left(\left\|g \chi_{k}\right\|_{L^{q^{\prime}(\cdot)}}\right)\right)^{p_{2}(1+\varepsilon)}\right)^{1 / p_{2}(1+\varepsilon)}\right) \\
= & \left.C\|f\|_{\dot{K}_{q(\cdot)}^{\left.\alpha_{1}, p_{1}\right), \theta}\left(\mathbb{R}^{n}\right)}\|g\|_{\dot{K}_{q^{\prime}(\cdot)}^{\left.\alpha_{2}, p_{2}\right), \theta}}\right)
\end{aligned}
$$

\section{Boundedness of sublinear operators}

In this section, we show that sublinear operators are bounded on $\dot{K}_{q(\cdot)}^{\alpha, p), \theta}\left(\mathbb{R}^{n}\right)$. Hernandez, $\mathrm{Li}, \mathrm{Lu}$ and Yang $[9,24,26]$ have proved that if a sublinear operator $T$ is bounded on $L^{p}\left(\mathbb{R}^{n}\right)$ and satisfies the size condition

$$
|T f(x)| \leq C \int_{\mathbb{R}^{n}}|x-y|^{-n}|f(y)| d y, \quad x \notin \operatorname{spt} f
$$

for all $f \in L^{1}\left(\mathbb{R}^{n}\right)$ with compact support then $T$ is bounded on the homogeneous Herz space $\dot{K}_{q}^{\alpha, p}$ and on the non-homogeneous Herz space $K_{q}^{\alpha, p}$. The condition (11) is satisfied by many interesting operators in harmonic analysis, such as Calderón-Zygmund operators, Carleson's maximal operator, the Hardy-Littlewood maximal operator, Fefferman's singular multipliers, Fefferman's singular integrals, Ricci-Stein's oscillatory singular integrals, and the Bochner-Riesz means (for details see [25, 34]).

Theorem 4.1 Let $1<p<\infty, q(\cdot) \in \mathcal{P}_{0, \infty}\left(\mathbb{R}^{n}\right)$ such that $-n / q(0)<\alpha<n / q^{\prime}(0)$ and $-n / q_{\infty}<$ $\alpha<n / q_{\infty}^{\prime}$. Suppose that $T$ is a sublinear operator and bounded on $L^{q(\cdot)}\left(\mathbb{R}^{n}\right)$ satisfying the size condition (10). Then $T$ is bounded on $\dot{K}_{q(\cdot)}^{\alpha, p), \theta}\left(\mathbb{R}^{n}\right)$.

Proof Since $T$ is sublinear, we have for every $f \in \dot{K}_{q(\cdot)}^{\alpha, p), \theta}\left(\mathbb{R}^{n}\right)$

$$
\begin{aligned}
\| T f & \|_{\dot{K}_{q(\cdot)}^{\alpha, p), \theta}\left(\mathbb{R}^{n}\right)} \\
= & \sup _{\varepsilon>0}\left(\varepsilon^{\theta} \sum_{k \in \mathbb{Z}} 2^{k \alpha p(1+\varepsilon)}\left\|\chi_{k} T f\right\|_{L^{q(\cdot)}\left(\mathbb{R}^{n}\right)}^{p(1+\varepsilon)}\right)^{\frac{1}{p(1+\varepsilon)}} \\
\leq & \sup _{\varepsilon>0}\left(\varepsilon^{\theta} \sum_{k \in \mathbb{Z}} 2^{k \alpha p(1+\varepsilon)}\left(\sum_{l=-\infty}^{\infty}\left\|\chi_{k} T\left(f \chi_{l}\right)\right\|_{L^{q(\cdot)}\left(\mathbb{R}^{n}\right)}^{p(1+\varepsilon)}\right)\right)^{\frac{1}{p(1+\varepsilon)}} \\
\leq & c \sup _{\varepsilon>0}\left(\varepsilon^{\theta} \sum_{k \in \mathbb{Z}} 2^{k \alpha p(1+\varepsilon)}\left(\sum_{l=-\infty}^{k-2}\left\|\chi_{k} T\left(f \chi_{l}\right)\right\|_{L^{q(\cdot)}\left(\mathbb{R}^{n}\right)}\right)^{p(1+\varepsilon)}\right)^{\frac{1}{p(1+\varepsilon)}} \\
& +c \sup _{\varepsilon>0}\left(\varepsilon^{\theta} \sum_{k \in \mathbb{Z}} 2^{k \alpha p(1+\varepsilon)}\left(\sum_{l=k-1}^{k+1}\left\|\chi_{k} T\left(f \chi_{l}\right)\right\|_{L^{q(\cdot)}\left(\mathbb{R}^{n}\right)}\right)^{p(1+\varepsilon)}\right)^{\frac{1}{p(1+\varepsilon)}} \\
& +c \sup _{\varepsilon>0}\left(\varepsilon^{\theta} \sum_{k \in \mathbb{Z}} 2^{k \alpha p(1+\varepsilon)}\left(\sum_{l=k+2}^{\infty}\left\|\chi_{k} T\left(f \chi_{l}\right)\right\|_{L^{q(\cdot)}\left(\mathbb{R}^{n}\right)}\right)^{p(1+\varepsilon)}\right)^{\frac{1}{p(1+\varepsilon)}} \\
= & E_{1}+E_{2}+E_{3} .
\end{aligned}
$$


For $E_{2}$, using the $L^{q(\cdot)}\left(\mathbb{R}^{n}\right)$ boundedness of $T$ we obtain

$$
\begin{aligned}
E_{2} & \leq c \sup _{\varepsilon>0}\left(\varepsilon^{\theta} \sum_{k \in \mathbb{Z}} 2^{k \alpha p(1+\varepsilon)}\left(\sum_{l=k-1}^{k+1}\left\|T\left(f \chi_{l}\right)\right\|_{L^{q(\cdot)}\left(\mathbb{R}^{n}\right)}\right)^{p(1+\varepsilon)}\right)^{\frac{1}{p(1+\varepsilon)}} \\
& \leq c \sup _{\varepsilon>0}\left(\varepsilon^{\theta} \sum_{k \in \mathbb{Z}} 2^{k \alpha p(1+\varepsilon)}\left(\sum_{l=k-1}^{k+1}\left\|f \chi_{l}\right\|_{L^{q(\cdot)}\left(\mathbb{R}^{n}\right)}\right)^{p(1+\varepsilon)}\right)^{\frac{1}{p(1+\varepsilon)}} \\
& \leq c \sup _{\varepsilon>0}\left(\varepsilon^{\theta} \sum_{k \in \mathbb{Z}} 2^{k \alpha p(1+\varepsilon)}\left\|f \chi_{k}\right\|_{L^{q(\cdot)\left(\mathbb{R}^{n}\right)}}^{p(1+\varepsilon)}\right)^{\frac{1}{p(1+\varepsilon)}} \\
& =c\|f\|_{\dot{K}_{q(\cdot)}^{\alpha, p), \theta}\left(\mathbb{R}^{n}\right)^{.}}
\end{aligned}
$$

For $E_{1}$, we use the facts that, for each $k \in \mathbb{Z}$ and $l \leq k-2$ and a.e. $x \in R_{k}$, size condition (10) and Hölder's inequality imply

$$
\begin{aligned}
\left|T\left(f \chi_{l}\right)(x)\right| & \leq C \int_{R_{l}}|x-y|^{-n}|f(y)| d y \\
& \leq c 2^{-k n} \int_{R_{l}}|f(y)| d y \leq c 2^{-k n}\left\|f \chi_{l}\right\|_{L^{q(\cdot)}\left(\mathbb{R}^{n}\right)}\left\|\chi_{l}\right\|_{L^{q^{(} \cdot()}\left(\mathbb{R}^{n}\right)}
\end{aligned}
$$

Moreover, splitting $E_{1}$ by means of Minkowski's inequality we have

$$
\begin{aligned}
E_{1} \leq & c \sup _{\varepsilon>0}\left(\varepsilon^{\theta} \sum_{k=-\infty}^{-1} 2^{k \alpha p(1+\varepsilon)}\left(\sum_{l=-\infty}^{k-2}\left\|\chi_{k} T\left(f \chi_{l}\right)\right\|_{L^{q(\cdot)}\left(\mathbb{R}^{n}\right)}\right)^{p(1+\varepsilon)}\right)^{\frac{1}{p(1+\varepsilon)}} \\
& +\quad \sup _{\varepsilon>0}\left(\varepsilon^{\theta} \sum_{k=0}^{\infty} 2^{k \alpha p(1+\varepsilon)}\left(\sum_{l=-\infty}^{k-2}\left\|\chi_{k} T\left(f \chi_{l}\right)\right\|_{L^{q(\cdot)}\left(\mathbb{R}^{n}\right)}\right)^{p(1+\varepsilon)}\right)^{\frac{1}{p(1+\varepsilon)}} \\
:= & E_{11}+E_{12} .
\end{aligned}
$$

For $E_{11}$ by Lemma 2.2 we have

$$
2^{-k n}\left\|\chi_{k}\right\|_{L^{q(\cdot)}\left(\mathbb{R}^{n}\right)}\left\|\chi_{l}\right\|_{L^{q^{\prime}(\cdot)}\left(\mathbb{R}^{n}\right)} \leq c 2^{-k n} 2^{\left(\frac{k n}{q(0)}\right)} 2^{\left(\frac{l n}{q^{\prime}(0)}\right)} \leq c 2^{\frac{(l-k) n}{q^{\prime}(0)}}
$$

Applying (11) and (12) to $E_{11}$, we get

$$
\begin{aligned}
& E_{11} \leq c \sup _{\varepsilon>0}\left(\varepsilon^{\theta} \sum_{k=-\infty}^{-1} 2^{k \alpha p(1+\varepsilon)}\right. \\
& \left.\times\left(\sum_{l=-\infty}^{k-2}\left\|\chi_{k}\right\|_{L^{q(\cdot)}\left(\mathbb{R}^{n}\right)} 2^{-k n}\left\|f \chi_{l}\right\|_{L^{q(\cdot)}\left(\mathbb{R}^{n}\right)}\left\|\chi_{l}\right\|_{L^{q^{\prime}(\cdot)}\left(\mathbb{R}^{n}\right)}\right)^{p(1+\varepsilon)}\right)^{\frac{1}{p(1+\varepsilon)}} \\
& \leq c \sup _{\varepsilon>0}\left(\varepsilon^{\theta} \sum_{k=-\infty}^{-1}\left(\sum_{l=-\infty}^{k-2} 2^{\alpha l}\left\|f \chi_{l}\right\|_{L^{q(\cdot)}\left(\mathbb{R}^{n}\right)} 2^{b(l-k)}\right)^{p(1+\varepsilon)}\right)^{\frac{1}{p(1+\varepsilon)}}
\end{aligned}
$$


where $b:=\frac{n}{q^{\prime}(0)}-\alpha>0$. Then we use Hölder's inequality, Fubini's theorem for series and $2^{-p(1+\varepsilon)}<2^{-p}$ to obtain

$$
\begin{aligned}
& \leq \underset{\varepsilon>0}{\leq} \sup _{\varepsilon>0} \varepsilon^{\theta} \sum_{k=-\infty}^{-1}\left(\sum_{l=-\infty}^{k-2} 2^{\alpha p(1+\varepsilon) l}\left\|f \chi_{l}\right\|_{L^{q^{(\cdot)}\left(\mathbb{R}^{n}\right)}}^{p(1+\varepsilon)} 2^{b p(1+\varepsilon)(l-k) / 2}\right) \\
& \left.\times\left(\sum_{l=-\infty}^{k-2} 2^{b(p(1+\varepsilon))^{\prime}(l-k) / 2}\right)^{p(1+\varepsilon) /(p(1+\varepsilon))^{\prime}}\right)^{\frac{1}{p(1+\varepsilon)}} \\
& =c \sup _{\varepsilon>0}\left(\varepsilon^{\theta} \sum_{k=-\infty}^{-1} \sum_{l=-\infty}^{k-2} 2^{\alpha p(1+\varepsilon) l}\left\|f \chi_{l}\right\|_{L^{q(\cdot)}\left(\mathbb{R}^{n}\right)}^{p(1+\varepsilon)} 2^{b p(1+\varepsilon)(l-k) / 2}\right)^{\frac{p}{p(1+\varepsilon)}} \\
& =c \sup _{\varepsilon>0}\left(\varepsilon^{\theta} \sum_{l=-\infty}^{-1} 2^{\alpha p(1+\varepsilon) l}\left\|f \chi_{l}\right\|_{L^{q(\cdot)}\left(\mathbb{R}^{n}\right)}^{p(1+\varepsilon)} \sum_{k=l+2}^{-1} 2^{b p(1+\varepsilon)(l-k) / 2}\right)^{\frac{1}{p(1+\varepsilon)}} \\
& <c \sup _{\varepsilon>0}\left(\varepsilon^{\theta} \sum_{l=-\infty}^{-1} 2^{\alpha p(1+\varepsilon) l}\left\|f \chi_{l}\right\|_{L^{q(\cdot)}\left(\mathbb{R}^{n}\right)}^{p(1+\varepsilon)} \sum_{k=l+2}^{-1} 2^{b p(l-k) / 2}\right)^{\frac{1}{p(1+\varepsilon)}} \\
& \leq c \sup _{\varepsilon>0}\left(\varepsilon^{\theta} \sum_{l \in \mathbb{Z}} 2^{\alpha p(1+\varepsilon) l}\left\|f \chi_{l}\right\|_{L^{q(\cdot)}\left(\mathbb{R}^{n}\right)}^{p(1+\varepsilon)}\right)^{\frac{1}{p(1+\varepsilon)}} \\
& \leq c\|f\|_{\dot{K}_{q(\cdot)}^{\alpha, p), \theta}\left(\mathbb{R}^{n}\right)^{\cdot}} \\
& \leq
\end{aligned}
$$

Now for $E_{12}$ using Minkowski's inequality we have

$$
\begin{aligned}
E_{12} \leq & c \sup _{\varepsilon>0}\left(\varepsilon^{\theta} \sum_{k=0}^{\infty} 2^{k \alpha p(1+\varepsilon)}\left(\sum_{l=-\infty}^{-1}\left\|\chi_{k} T\left(f \chi_{l}\right)\right\|_{L^{q(\cdot)}\left(\mathbb{R}^{n}\right)}\right)^{p(1+\varepsilon)}\right)^{\frac{1}{p(1+\varepsilon)}} \\
& +c \sup _{\varepsilon>0}\left(\varepsilon^{\theta} \sum_{k=0}^{\infty} 2^{k \alpha p(1+\varepsilon)}\left(\sum_{l=0}^{k-2}\left\|\chi_{k} T\left(f \chi_{l}\right)\right\|_{L^{q(\cdot)}\left(\mathbb{R}^{n}\right)}\right)^{p(1+\varepsilon)}\right)^{\frac{1}{p(1+\varepsilon)}} \\
:= & A_{1}+A_{2} .
\end{aligned}
$$

The estimate for $A_{2}$ follows in a similar manner to $E_{11}$ with $q^{\prime}(0)$ replaced by $q_{\infty}^{\prime}$ and using the fact that $\frac{n}{q_{\infty}^{\prime}}-\alpha>0$. For $A_{1}$ using Lemma 2.2 we have

$$
2^{-k n}\left\|\chi_{k}\right\|_{L^{q(\cdot)}\left(\mathbb{R}^{n}\right)}\left\|\chi_{l}\right\|_{L^{q^{\prime}(\cdot)}\left(\mathbb{R}^{n}\right)} \leq c 2^{-k n} 2^{\left(\frac{k n}{q \infty}\right)} 2^{\left(\frac{l n}{q^{\prime}(0)}\right)} \leq c 2^{\left(\frac{-k n}{q_{\infty}^{\prime}}\right)} 2^{\left(\frac{l n}{q^{\prime}(0)}\right)} .
$$

Now using (11) and (14) and the fact that $\alpha-\frac{n}{q_{\infty}^{\prime}}<0$ we have

$$
\begin{aligned}
A_{1} \leq & \sup _{\varepsilon>0}\left(\varepsilon^{\theta} \sum_{k=0}^{\infty} 2^{k \alpha p(1+\varepsilon)}\left(\sum_{l=-\infty}^{-1}\left\|\chi_{k} T f\left(\chi_{l}\right)\right\|_{L^{q(\cdot)}\left(\mathbb{R}^{n}\right)}\right)^{p(1+\varepsilon)}\right)^{1 / p(1+\varepsilon)} \\
\leq & c \sup _{\varepsilon>0}\left(\varepsilon^{\theta} \sum_{k=0}^{\infty} 2^{k \alpha p(1+\varepsilon)}\right. \\
& \left.\times\left(\sum_{l=-\infty}^{-1} 2^{-k n} 2^{\left(k n / q_{\infty}\right)} 2^{\left(\ln / q^{\prime}(0)\right)}\left\|f\left(\chi_{l}\right)\right\|_{L^{q(\cdot)}\left(\mathbb{R}^{n}\right)}\right)^{p(1+\varepsilon)}\right)^{1 / p(1+\varepsilon)}
\end{aligned}
$$


Naris et al. Journal of Inequalities and Applications

(2020) 2020:1

Page 9 of 13

$$
\begin{aligned}
\leq & \underset{\varepsilon>0}{c}\left(\varepsilon^{\theta} \sum_{k=0}^{\infty} 2^{k \alpha p(1+\varepsilon)}\right. \\
& \left.\times\left(\sum_{l=-\infty}^{-1} 2^{\left(-k n / q_{\infty}^{\prime}\right)} 2^{\left(l n / q^{\prime}(0)\right)}\left\|f\left(\chi_{l}\right)\right\|_{L^{q(\cdot)}\left(\mathbb{R}^{n}\right)}\right)^{p(1+\varepsilon)}\right)^{1 / p(1+\varepsilon)} \\
\leq & \left.c \sup _{\varepsilon>0}\left(\varepsilon^{\theta} \sum_{k=0}^{\infty} 2^{\left(k \alpha-k n / q_{\infty}^{\prime}\right) p(1+\varepsilon)}\right)^{p(1+\varepsilon)}\right)^{1 / p(1+\varepsilon)} \\
& \left.\times\left(\sum_{l=-\infty}^{-1} 2^{\left(\ln / q^{\prime}(0)\right)}\left\|f\left(\chi_{l}\right)\right\|_{L^{q(\cdot)}\left(\mathbb{R}^{n}\right)}\right)^{p(1+\varepsilon)}\right)^{1 / p(1+\varepsilon)} \\
\leq & c \sup _{\varepsilon>0}\left(\varepsilon^{\theta}\left(\sum_{l=-\infty}^{-1} 2^{\left(l n / q^{\prime}(0)\right)}\left\|f\left(\chi_{l}\right)\right\|_{L^{q(\cdot)}\left(\mathbb{R}^{n}\right)}\right)^{p(1+\varepsilon)}\right)^{1 / p(1+\varepsilon)} \\
\leq & c \sup _{\varepsilon>0}\left(\varepsilon ^ { \theta } \left(\sum_{l=-\infty}^{-1} 2^{l \alpha}\left\|f\left(\chi_{l}\right)\right\|_{L^{q(\cdot)}\left(\mathbb{R}^{n}\right)^{\left(l n / q^{\prime}(0)-l \alpha\right)}}\right.\right.
\end{aligned}
$$

Now applying Hölder's inequality and using the fact that $\frac{n}{q^{\prime}(0)}-\alpha>0$ we have

$$
\begin{aligned}
A_{1} \leq & c \sup _{\varepsilon>0}\left(\varepsilon^{\theta}\left(\sum_{l=-\infty}^{-1} 2^{l \alpha p(1+\varepsilon)}\left\|f\left(\chi_{l}\right)\right\|_{L^{q(\cdot)}}^{p(1+\varepsilon)}\right)\right. \\
& \left.\times\left(\sum_{l=-\infty}^{-1} 2^{l\left(n / q^{\prime}(0)-\alpha\right)(p(1+\varepsilon))^{\prime}}\right)^{p(1+\varepsilon) /(p(1+\varepsilon))^{\prime}}\right)^{1 / p(1+\varepsilon)} \\
\leq & c \sup _{\varepsilon>0}\left(\varepsilon^{\theta}\left(\sum_{l \in \mathbb{Z}} 2^{l \alpha p(1+\varepsilon)}\left\|f\left(\chi_{l}\right)\right\|_{L^{q(\cdot)\left(\mathbb{R}^{n}\right)}}^{p(1+\varepsilon)}\right)\right)^{1 / p(1+\varepsilon)} \\
\leq & c\|f\|_{K_{q(\cdot)}^{\alpha, p), \theta}\left(\mathbb{R}^{n}\right)}
\end{aligned}
$$

Next, we estimate $E_{3}$. For each $k \in \mathbb{Z}$ and $l \geq k+2$ and a.e. $x \in R_{k}$; the size condition (11) and Hölder's inequality imply

$$
\begin{aligned}
\left|T\left(f \chi_{l}\right)(x)\right| & \leq C \int_{R_{l}}|x-y|^{-n}|f(y)| d y \\
& \leq c 2^{-\ln } \int_{R_{l}}|f(y)| d y \leq c 2^{-\ln }\left\|f \chi_{l}\right\|_{L^{q(\cdot)}\left(\mathbb{R}^{n}\right)}\left\|\chi_{l}\right\|_{L^{q^{\prime}(\cdot)}\left(\mathbb{R}^{n}\right)^{.}}
\end{aligned}
$$

Similar to $E_{1}$, splitting $E_{3}$ by means of Minkowski's inequality we have

$$
\begin{aligned}
E_{3} \leq & c \sup _{\varepsilon>0}\left(\varepsilon^{\theta} \sum_{k=-\infty}^{-1} 2^{k \alpha p(1+\varepsilon)}\left(\sum_{l=k+2}^{\infty}\left\|\chi_{k} T\left(f \chi_{l}\right)\right\|_{L^{q(\cdot)\left(\mathbb{R}^{n}\right)}}\right)^{p(1+\varepsilon)}\right)^{\frac{1}{p(1+\varepsilon)}} \\
& +c \sup _{\varepsilon>0}\left(\varepsilon^{\theta} \sum_{k=0}^{\infty} 2^{k \alpha p(1+\varepsilon)}\left(\sum_{l=k+2}^{\infty}\left\|\chi_{k} T\left(f \chi_{l}\right)\right\|_{L^{q(\cdot)}\left(\mathbb{R}^{n}\right)}\right)^{p(1+\varepsilon)}\right)^{\frac{1}{p(1+\varepsilon)}} \\
:= & E_{31}+E_{32} .
\end{aligned}
$$


Naris et al. Journal of Inequalities and Applications

(2020) $2020: 1$

Page 10 of 13

For $E_{32}$ Lemma 2.2 yields

$$
2^{-\ln }\left\|\chi_{k}\right\|_{L^{q(\cdot)}\left(\mathbb{R}^{n}\right)}\left\|\chi_{l}\right\|_{L^{q^{\prime}(\cdot)}\left(\mathbb{R}^{n}\right)} \leq c 2^{-\ln } 2^{\left(\frac{k n}{q_{\infty}}\right)} 2^{\left(\frac{\ln }{q_{\infty}^{\prime}}\right)} \leq c 2^{\frac{(k-l) n}{q \infty}}
$$

Using (15) and (16) for $E_{32}$, we get

$$
\begin{aligned}
E_{32} \leq & c \sup _{\varepsilon>0}\left(\varepsilon^{\theta} \sum_{k=0}^{\infty} 2^{k \alpha p(1+\varepsilon)}\right. \\
& \left.\times\left(\sum_{l=k+2}^{\infty}\left\|\chi_{k}\right\|_{L^{q(\cdot)\left(\mathbb{R}^{n}\right)}} 2^{-l n}\left\|f \chi_{l}\right\|_{L^{q(\cdot)}\left(\mathbb{R}^{n}\right)}\left\|\chi_{l}\right\|_{L^{q^{\prime}(\cdot)\left(\mathbb{R}^{n}\right)}}\right)^{p(1+\varepsilon)}\right)^{\frac{1}{p(1+\varepsilon)}} \\
\leq & \sup _{\varepsilon>0}\left(\varepsilon^{\theta} \sum_{k=0}^{\infty}\left(\sum_{l=k+2}^{\infty} 2^{\alpha l}\left\|f \chi_{l}\right\|_{L^{q(\cdot)}\left(\mathbb{R}^{n}\right)} 2^{d(k-l)}\right)^{p(1+\varepsilon)}\right)^{\frac{1}{p(1+\varepsilon)}}
\end{aligned}
$$

where $d:=\frac{n}{q_{\infty}}+\alpha>0$. Then we use Hölder's inequality, Fubini's theorem for series and $2^{-p(1+\varepsilon)}<2^{-p}$ to obtain

$$
\begin{aligned}
\leq & c \sup _{\varepsilon>0}\left(\varepsilon^{\theta} \sum_{k=0}^{\infty}\left(\sum_{l=k+2}^{\infty} 2^{\alpha p(1+\varepsilon) l}\left\|f \chi_{l}\right\|_{L^{q(\cdot)}\left(\mathbb{R}^{n}\right)}^{p(1+\varepsilon)} 2^{d p(1+\varepsilon)(k-l) / 2}\right)\right. \\
& \left.\times\left(\sum_{l=k+2}^{\infty} 2^{d(p(1+\varepsilon))^{\prime}(k-l) / 2}\right)^{p(1+\varepsilon) /(p(1+\varepsilon))^{\prime}}\right)^{\frac{1}{p(1+\varepsilon)}} \\
= & \sup _{\varepsilon>0}\left(\varepsilon^{\theta} \sum_{k=0}^{\infty} \sum_{l=k+2}^{\infty} 2^{\alpha p(1+\varepsilon) l}\left\|f \chi_{l}\right\|_{L^{q(\cdot)}\left(\mathbb{R}^{n}\right)}^{p(1+\varepsilon)} 2^{d p(1+\varepsilon)(k-l) / 2}\right)^{\frac{1}{p(1+\varepsilon)}} \\
= & \sup _{\varepsilon>0}\left(\varepsilon^{\theta} \sum_{l=0}^{\infty} 2^{\alpha p(1+\varepsilon) l}\left\|f \chi_{l}\right\|_{L^{q(\cdot)}\left(\mathbb{R}^{n}\right)}^{p(1+\varepsilon)} \sum_{k=0}^{l-2} 2^{d p(1+\varepsilon)(k-l) / 2}\right)^{\frac{1}{p(1+\varepsilon)}} \\
< & c \sup _{\varepsilon>0}\left(\varepsilon^{\theta} \sum_{l \in \mathbb{Z}} 2^{\alpha p(1+\varepsilon) l}\left\|f \chi_{l}\right\|_{L^{q(\cdot)}\left(\mathbb{R}^{n}\right)}^{p(1+\varepsilon)} \sum_{k=-\infty}^{l-2} 2^{d p(k-l) / 2}\right)^{\frac{1}{p(1+\varepsilon)}} \\
= & c \sup _{\varepsilon>0}\left(\varepsilon^{\theta} \sum_{l \in \mathbb{Z}} 2^{\alpha p(1+\varepsilon) l}\left\|f \chi_{l}\right\|_{L^{q(\cdot)}\left(\mathbb{R}^{n}\right)}^{p(1+\varepsilon)}\right)^{\frac{1}{p(1+\varepsilon)}} \\
\leq & c\|f\|_{K_{q(\cdot)}^{\alpha, p), \theta}\left(\mathbb{R}^{n}\right)^{\cdot}}
\end{aligned}
$$

Now for $E_{31}$ using Minkowski's inequality we have

$$
\begin{aligned}
E_{31} \leq & c \sup _{\varepsilon>0}\left(\varepsilon^{\theta} \sum_{k=-\infty}^{-1} 2^{k \alpha p(1+\varepsilon)}\left(\sum_{l=k+2}^{-1}\left\|\chi_{k} T\left(f \chi_{l}\right)\right\|_{L^{q(\cdot)}\left(\mathbb{R}^{n}\right)}\right)^{p(1+\varepsilon)}\right)^{\frac{1}{p(1+\varepsilon)}} \\
& +c \sup _{\varepsilon>0}\left(\varepsilon^{\theta} \sum_{k=-\infty}^{-1} 2^{k \alpha p(1+\varepsilon)}\left(\sum_{l=0}^{\infty}\left\|\chi_{k} T\left(f \chi_{l}\right)\right\|_{L^{q(\cdot)}\left(\mathbb{R}^{n}\right)}\right)^{p(1+\varepsilon)}\right)^{\frac{1}{p(1+\varepsilon)}} \\
:= & B_{1}+B_{2} .
\end{aligned}
$$


Naris et al. Journal of Inequalities and Applications

(2020) 2020:1

Page 11 of 13

The estimate for $B_{1}$ follows in a similar manner to $E_{32}$ with $q_{\infty}$ replaced by $q(0)$ and using the fact that $\frac{n}{q(0)}+\alpha>0$. For $B_{2}$ using Lemma 2.2 we have

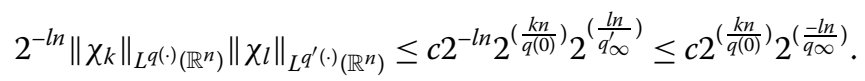

Now using (15) and (17) and the fact that $\alpha+\frac{n}{q(0)}>0$ we have

$$
\begin{aligned}
& B_{2} \leq \sup _{\varepsilon>0}\left(\varepsilon^{\theta} \sum_{k=-\infty}^{-1} 2^{k \alpha p(1+\varepsilon)}\left(\sum_{l=0}^{\infty}\left\|\chi_{k} T f\left(\chi_{l}\right)\right\|_{L^{q(\cdot)\left(\mathbb{R}^{n}\right)}}\right)^{p(1+\varepsilon)}\right)^{1 / p(1+\varepsilon)} \\
& \leq c \sup _{\varepsilon>0}\left(\varepsilon^{\theta} \sum_{k=-\infty}^{-1} 2^{k \alpha p(1+\varepsilon)}\right.
\end{aligned}
$$

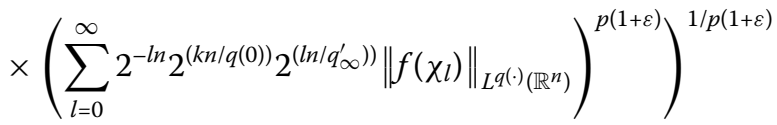

$$
\begin{aligned}
& \leq c \sup _{\varepsilon>0}\left(\varepsilon^{\theta} \sum_{k=-\infty}^{-1} 2^{k \alpha p(1+\varepsilon)}\right. \\
& \left.\times\left(\sum_{l=0}^{\infty} 2^{(k n / q(0))} 2^{-\left(\ln / q_{\infty}\right)}\left\|f\left(\chi_{l}\right)\right\|_{L^{q(\cdot)}\left(\mathbb{R}^{n}\right)}\right)^{p(1+\varepsilon)}\right)^{1 / p(1+\varepsilon)} \\
& \leq c \sup _{\varepsilon>0}\left(\varepsilon^{\theta} \sum_{k=-\infty}^{-1} 2^{k(\alpha+n / q(0)) p(1+\varepsilon)}\right. \\
& \left.\times\left(\sum_{l=0}^{\infty} 2^{-\left(\ln / q_{\infty}\right)}\left\|f\left(\chi_{l}\right)\right\|_{L^{q(\cdot)}\left(\mathbb{R}^{n}\right)}\right)^{p(1+\varepsilon)}\right)^{1 / p(1+\varepsilon)} \\
& \leq c \sup _{\varepsilon>0}\left(\varepsilon^{\theta}\left(\sum_{l=0}^{\infty} 2^{-\left(\ln / q_{\infty}\right)}\left\|f\left(\chi_{l}\right)\right\|_{L^{q(\cdot)}\left(\mathbb{R}^{n}\right)}\right)^{p(1+\varepsilon)}\right)^{1 / p(1+\varepsilon)} \\
& \leq c \sup _{\varepsilon>0}\left(\varepsilon^{\theta}\left(\sum_{l=0}^{\infty} 2^{l \alpha}\left\|f\left(\chi_{l}\right)\right\|_{L^{q(\cdot)}\left(\mathbb{R}^{n}\right)} 2^{-l\left(n / q_{\infty}+\alpha\right)}\right)^{p(1+\varepsilon)}\right)^{1 / p(1+\varepsilon)} .
\end{aligned}
$$

Now applying Hölder's inequality and using the fact that $\frac{n}{q_{\infty}}+\alpha>0$ we have

$$
\begin{aligned}
& B_{2} \leq c \sup _{\varepsilon>0}\left(\varepsilon^{\theta}\left(\sum_{l=0}^{\infty} 2^{l \alpha p(1+\varepsilon)}\left\|f\left(\chi_{l}\right)\right\|_{L^{q(\cdot)}}^{p(1+\varepsilon)}\right)\right. \\
&\left.\times\left(\sum_{l=0}^{\infty} 2^{-l(n / q \infty+\alpha)(p(1+\varepsilon))^{\prime}}\right)^{p(1+\varepsilon) /(p(1+\varepsilon))^{\prime}}\right)^{1 / p(1+\varepsilon)} \\
& \leq c \sup _{\varepsilon>0}\left(\varepsilon^{\theta} \sum_{l \in \mathbb{Z}} 2^{l \alpha p(1+\varepsilon)}\left\|f\left(\chi_{l}\right)\right\|_{L^{q(\cdot)\left(\mathbb{R}^{n}\right)}}^{p(1+\varepsilon)}\right)^{1 / p(1+\varepsilon)} \\
& \leq c\|f\|_{\dot{K}_{q(\cdot)}^{\alpha, p), \theta}\left(\mathbb{R}^{n}\right)^{.}}
\end{aligned}
$$


Combining the estimates for $E_{1}, E_{2}$ and $E_{3}$ yields

$$
\|T f\|_{\dot{K}_{q(\cdot)}^{\alpha, p), \theta}\left(\mathbb{R}^{n}\right)} \leq c\|f\|_{\dot{K}_{q(\cdot)}^{\alpha, p), \theta}\left(\mathbb{R}^{n}\right)^{\prime}}
$$

which ends the proof.

\section{Acknowledgements}

Not applicable.

\section{Funding}

The research of H. Rafeiro was supported by a Research Start-Up Grant of United Arab Emirates University, Al Ain, United Arab Emirates via Grant No. G00002994.

\section{Availability of data and materials}

Not applicable.

\section{Competing interests}

The authors declare that there are no competing interests.

\section{Authors' contributions}

There was an equal amount of contributions from all three authors. All authors read and approved the final manuscript.

\section{Author details}

'Department of Mathematics and Statistics, Riphah International University, Islamabad, Pakistan. ${ }^{2}$ College of Sciences, Department of Mathematical Sciences, United Arab Emirates University, Al Ain, United Arab Emirates.

\section{Publisher's Note}

Springer Nature remains neutral with regard to jurisdictional claims in published maps and institutional affiliations.

Received: 16 May 2019 Accepted: 6 December 2019 Published online: 02 January 2020

\section{References}

1. Almeida, A., Drihem, D.: Maximal, potential and singular type operators on Herz spaces with variable exponents. J. Math. Anal. Appl. 394(2), 781-795 (2012)

2. Castillo, R.E., Rafeiro, H.: An Introductory Course in Lebesgue Spaces. Springer, Berlin (2016)

3. Cruz-Uribe, D., Fiorenza, A.: Variable Lebesgue Space: Foundations and Harmonic Analysis. Birkhäuser, Basel (2013)

4. Diening, L., Harjulehto, P., Hästö, P., Růžička, M.: Lebesgue and Sobolev Spaces with Variable Exponents. Lecture Notes in Math., vol. 2017. Springer, Berlin (2011)

5. Feichtinger, H.G., Weisz, F.: Herz spaces and summability of Fourier transforms. Math. Nachr. 281(3), 309-324 (2008)

6. Fiorenza, A., Gupta, B., Jain, P.: The maximal theorem for weighted grand Lebesgue spaces. Stud. Math. 188(2), 123-133 (2008)

7. Grafakos, L., Li, X., Yang, D.: Bilinear operators on Herz-type Hardy spaces. Trans. Am. Math. Soc. 350(3), 1249-1275 (1998)

8. Greco, L., Iwaniec, T., Sbordone, C.: Inverting the $p$-harmonic operator. Manuscr. Math. 92(2), 249-258 (1997)

9. Hernandez, E., Yang, D.: Interpolation of Herz spaces and application. Math. Nachr. 205(1), 69-87 (1999)

10. Herz, C.: Lipschitz spaces and Bernstein's theorem on absolutely convergent Fourier transform. J. Math. Mech. 18, 283-323 (1968)

11. Iwaniec, T., Sbordone, C.: On the integrability of the Jacobian under minimal hypotheses. Arch. Ration. Mech. Anal. $119(2), 129-143(1992)$

12. Izuki, M.: Boundedness of vector-valued sublinear operators on Herz-Morrey spaces with variable exponent. Math. Sci. Res. J. 13(10), 243-253 (2009)

13. Izuki, M.: Boundedness of sublinear operators on Herz spaces with variable exponent and application to wavelet characterization. Anal. Math. 13(36), 33-50 (2010)

14. Johnson, R.: Temperature, Riesz potenial and the Lipschitz spaces of Herz. Proc. Lond. Math. Soc. 27(2), 290-316 (1973)

15. Johnson, R.: Lipschitz spaces, Littlewood-Paley spaces and convoluteurs. Proc. Lond. Math. Soc. 29(1), 127-141 (1974)

16. Kokilashvili, V.: Boundedness criteria for singular integrals in weighted grand Lebesgue spaces. J. Math. Sci. (N.Y.) 170(1), 20-33 (2010)

17. Kokilashvili, V., Meskhi, A.: A note on the boundedness of the Hilbert transform in weighted grand Lebesgue spaces Georgian Math. J. 16(3), 547-551 (2009)

18. Kokilashvili, V., Meskhi, A.: Trace inequalities for fractional integrals in grand Lebesque spaces. Stud. Math. 210(2), 159-176 (2012)

19. Kokilashvili, V., Meskhi, A.: Potentials with product kernels in grand Lebesgue spaces: one-weight criteria. Lith. Math. J. 23(1), 27-39 (2013)

20. Kokilashvili, V., Meskhi, A., Rafeiro, H., Samko, S.: Integral Operators in Non-Standard Function Spaces, vol. 1. Oper. Theory Adv. Appl., vol. 248. Birkhäuser, Basel (2016) 
21. Kokilashvili, V., Meskhi, A., Rafeiro, H., Samko, S.: Integral Operators in Non-Standard Function Spaces, vol. 2. Oper. Theory Adv. Appl., vol. 249. Birkhäuser, Basel (2016)

22. Komori, Y.: Notes on singular integrals on some inhomogeneous Herz spaces. Taiwan. J. Math. 8(3), 547-556 (2004)

23. Kováčik, O., Rákosník, J.: On spaces $L^{p(x)}$ and $W^{k, p(x)}$. Czechoslov. Math. J. 41(4), 592-618 (1991)

24. Li, X., Yang, D.: Boundedness of some sublinear operators on Herz spaces. III. J. Math. 40, 484-501 (1996)

25. Lu, G., Lu, S., Yang, D.: Singular integrals and commutators on homogeneous groups. Anal. Math. 28(2), 103-134 (2002)

26. Lu, S., Yang, S.: The decomposition of the weighted Herz spaces and its application. Sci. China Ser. A 38, 147-158 (1995)

27. Meskhi, A.: Weighted criteria for the Hardy transform under the $B_{p}$ condition in grand Lebesgue spaces and some application. J. Math. Sci. (N.Y.) 178(6), 622-636 (2011)

28. Meskhi, A.: Criteria for the boundedness of potential operators in grand Lebesgue spaces. Proc. A. Razmadze Math. Inst. 169, 119-132 (2015)

29. Meskhi, A., Rafeiro, H., Zaighum, M.A.: Central Calderón-Zygmund operators on Herz-type Hardy spaces of variable smoothness and integrability. Ann. Funct. Anal. 9(3), 310-321 (2018)

30. Rădulescu, V.D., Repovš, D.: Partial Differential Equations with Variable Exponents: Variational Methods and Qualitative Analysis. Chapman \& Hall/CRC, Boca Raton (2015)

31. Rafeiro, H., Samko, S., Umarkhadzhiev, S.: Grand Lebesgue sequence spaces. Georgian Math. J. 25(2), 291-302 (2018)

32. Ruzicka, M.: Electroreological Fluids: Modeling and Mathematical Theory. Lecture Notes in Math., vol. 1748. Springer, Berlin (2000)

33. Samko, S.G.: Variable exponents Herz space. Mediterr. J. Math. 10(4), 2007-2025 (2013)

34. Soria, F., Weiss, G.: A remark on singular integrals and power weights. Indiana Univ. Math. J. 43(1), 187-204 (1994)

\section{Submit your manuscript to a SpringerOpen ${ }^{\circ}$ journal and benefit from:}

- Convenient online submission

- Rigorous peer review

- Open access: articles freely available online

- High visibility within the field

- Retaining the copyright to your article

Submit your next manuscript at $>$ springeropen.com 